\title{
COVID-19 pneumonia: relationship between inflammation assessed by whole-body FDG PET/CT and short-term clinical outcome
}

\author{
Matthieu Dietz ${ }^{1} \cdot$ Gilles Chironi $^{2} \cdot$ Yann-Erick Claessens $^{3} \cdot$ Ryan Lukas Farhad $^{4} \cdot$ Isabelle Rouquette $^{5}$. \\ Benjamin Serrano ${ }^{6}$. Valérie Nataf ${ }^{1}$. Florent Hugonnet ${ }^{1} \cdot$ Benoît Paulmier $^{1}$. Frédéric Berthier ${ }^{7}$. Olivia Keita-Perse ${ }^{8}$. \\ Francesco Giammarile ${ }^{9,10} \cdot$ Christophe Perrin ${ }^{4} \cdot$ Marc Faraggi $^{1}$ (D) $\cdot$ for the MONACOVID Group
}

Received: 28 May 2020 / Accepted: 19 July 2020 / Published online: 25 July 2020

(C) Springer-Verlag GmbH Germany, part of Springer Nature 2020

\begin{abstract}
Purpose [18F]-2-Fluoro-2-deoxy-D-glucose PET/CT (FDG PET/CT) is a sensitive and quantitative technic for detecting inflammatory process. Glucose uptake is correlated with an increased anaerobic glycolysis seen in activated inflammatory cells such as monocytes, lymphocytes, and granulocytes. The aim of the study was to assess the inflammatory status at the presumed peak of the inflammatory phase in non-critically ill patients requiring admission for COVID-19.

Methods Patients admitted with COVID-19 were prospectively enrolled. FDG PET/CT was performed from day 6 to day 14 of the onset of symptoms. Depending on FDG PET/CT findings, patients' profiles were classified as "inflammatory" or "low inflammatory." FDG PET/CT data were compared with chest CT evolution and short-term clinical outcome. All inflammatory sites were reported to screen potential extra-pulmonary tropism.

Results Thirteen patients were included. Maximum standardized uptake values ranged from 4.7 to 16.3 in lungs. All patients demonstrated increased mediastinal lymph nodes glucose uptake. Three patients $(23 \%)$ presented mild nasopharyngeal, two patients (15\%) bone marrow, and five patients (38\%) splenic mild increase in glucose uptake. No patient had significant digestive focal or segmental glucose uptake. There was no significant physiological myocardial glucose uptake in all patients except one. There was no correlation between PET lung inflammatory status and chest CT evolution or short-term clinical outcome.

Conclusion Inflammatory process at the presumed peak of the inflammatory phase in COVID-19 patients is obvious in FDG $\mathrm{PET} / \mathrm{CT}$ scans. Glucose uptake is heterogeneous and typically focused on lungs.

Trial registration NCT04441489. Registered 22 June 2020 (retrospectively registered).
\end{abstract}

Keywords COVID-19 · FDG PET/CT · Inflammation

This article is part of the Topical Collection on Infection and inflammation

Electronic supplementary material The online version of this article (https://doi.org/10.1007/s00259-020-04968-8) contains supplementary material, which is available to authorized users.

Marc Faraggi

marc.faraggi@chpg.mc

1 Nuclear Medicine Department, Centre Hospitalier Princesse Grace, 1 Avenue Pasteur, 98000 Monaco, Monaco

2 Check-up Unit, Centre Hospitalier Princesse Grace, Monaco, Monaco

3 Department of Emergency Medicine, Centre Hospitalier Princesse Grace, Monaco, Monaco

4 Pulmonary Department, Centre Hospitalier Princesse Grace, Monaco, Monaco
5 Department of Intensive Care Medicine, Centre Hospitalier Princesse Grace, Monaco, Monaco

6 Medical Physics Department, Centre Hospitalier Princesse Grace, Monaco, Monaco

7 Department of Biostatistics, Centre Hospitalier Princesse Grace, Monaco, Monaco

8 Infection disease Department, Centre Hospitalier Princesse Grace, Monaco, Monaco

9 Division of Human Health, International Atomic Energy Agency, Vienna, Austria

10 Centre Leon Berard, Lyon, France 


\section{Introduction}

In the history of coronavirus disease 2019 (COVID-19) caused by severe acute respiratory syndrome coronavirus- 2 (SARS-CoV-2), a minority of patients develop symptoms of acute respiratory distress syndrome (ARDS) that may require invasive mechanical ventilation and threaten short-term outcome. The mean delay from the onset of symptoms to ARDS is 10 days [1-3] and can occur even with very low viral load [4]. Since the beginning of the outbreak, chest computed tomography (CT) scan has been reported as the cornerstone to assist diagnosis and early screening $[5,6]$. Repeated CT may also explain some of the secondary clinical worsening by revealing secondary infections or pulmonary embolism [7].

However, unexpected respiratory distress may occur without any significant changes on CT images, once pulmonary embolism or secondary infection is discarded. One possible reason could be lung inflammation, involving the cell-mediated immunity and cytokine storms [1]. This inflammatory cascade sequence can lead to fatal immune-mediated injuries . CT scan displays suggestive images of alveolar and interstitial edema and its extent. Those might be part of inflammation consequences [8] but cannot explain the underlying mechanism (active inflammatory cell infiltration or not) or quantify the intensity of the inflammatory process. Furthermore, CT images abnormalities may persist a long time after clinical symptom resolution and do not reflect current active inflammation [9]. Imaging the active inflammation could be very useful for a better understanding of this particular phase of the infection. Combined [18F]-2-fluoro-2-deoxy-D-glucose positron emission tomographic imaging with fused CT (FDG PET/CT) is fitted for this purpose. FDG uptake is correlated with an increased anaerobic glycolysis seen in activated inflammatory cells such as monocytes, lymphocytes, and granulocytes [10]. Chefer et al. used FDG PET/CT to investigate the host lymphoid tissue response developing in nonhuman primates after exposure to Middle East respiratory syndrome (MERS) caused by a coronavirus, MERS-CoV [11]. FDG PET/CT has also been used to evaluate anti-inflammatory treatments by measuring changes in pulmonary inflammation [12]. FDG $\mathrm{PET} / \mathrm{CT}$ is able to quantitatively investigate the entire body at the cellular level because FDG uptake is cell-mediated.

The aim of the study was to evaluate the inflammatory status at the presumed peak of the inflammatory phase in non-critically ill patients requiring an hospitalization for COVID-19.

\section{Methods}

\section{Patients}

Patients presenting with COVID-19 were prospectively enrolled in this study carried out at the Centre Hospitalier
Princesse Grace of Monaco from March 27th to May 3rd, 2020. Patients were included if COVID-19 was confirmed according to the WHO guidance [13] by a positive result of RT-PCR assay of nasal and pharyngeal swabs, if they were hospitalized during the time period from day 6 to day 14 of the onset of symptoms, and if their initial (on admission) chest CT scan presented ground-glass opacities (GGO) or consolidation. Patients could not be included if their medical condition was unstable or precluded a safe transfer to the nuclear medicine department, if they were under mechanical ventilation (either non-invasive or invasive), if they were initially referred to the critical care unit, or in case of a pregnancy.

The institutional review board for human studies approved the protocol and a written consent was obtained from the subjects. Personal protective equipment (PPE) was available for the staff, and all measures to ensure strict infection prevention were observed according to established guidance [14].

\section{Chest CT scan and FDG PET/CT}

All patients had an initial chest CT scan for diagnosis on admission and a second chest CT scan for follow-up concomitant with a FDG PET/CT between day 6 and day 14 after the onset of symptoms. A full description of the technical considerations and dosimetry is provided in Table E1 in the supplementary data online.

FDG PET/CT and chest CT images were independently analyzed by 2 nuclear medicine physicians (MF and MD) with several years of experience in reading cardiothoracic imaging, on commercially available software (Syngo.via, Siemens version VB30). These physicians were blinded to any biological or clinical data. In case of disagreement, a consensus reading was performed.

\section{Chest CT images interpretation}

The degree of involvement of GGO and consolidation, expressed as a percentage of the total pulmonary volume according to previous studies $[9,15]$, was visually evaluated on the initial chest $\mathrm{CT}$ scan (on admission) and on the second chest CT scan concomitant with the FDG PET/CT. Based on the changes between the two chest CTs, patients were classified as follows:

- CT worsening: increase of CT abnormalities extent > $20 \%$ or additional GGO or consolidation. Conversion of GGO to consolidation was not considered as an additional finding.

- CT stability: changes of CT abnormalities extent $\leq 20 \%$ without additional GGO or consolidation.

- CT improvement: decrease of CT abnormalities extent > $20 \%$ without additional GGO or consolidation. 


\section{FDG PET/CT images' interpretation}

The amount of FDG pathological uptake was expressed by maximum signal intensity standardized uptake values $\left(\mathrm{SUV}_{\max }\right)$. Hypermetabolic volumes (defined as the volume of pixels in the region of interest with $\mathrm{SUV}_{\max }$ value above 4) were computerized. Based on FDG PET/CT imaging, patients' profiles were classified as follows:

- Inflammatory: lungs hypermetabolic volume $\geq 50 \mathrm{ml}$ and/or $\mathrm{SUV}_{\max } \geq 7$

- Low inflammatory: lungs hypermetabolic volume < $50 \mathrm{ml}$ and/or $\mathrm{SUV}_{\max }<7$

The rationale for the selection of $\mathrm{SUV}_{\max }$ and hypermetabolic volume values is explained in the supplementary data online and in Table E2.

All inflammatory sites in the acquisition field from skull top to mid-thigh were also reported to screen SARS-CoV-2 potential extra-pulmonary tropism.

\section{Adjudication committee classification for patients' short-term clinical outcome}

An expert panel was launched to adjudicate patients' shortterm clinical outcome. The expert panel consisted of 3 physicians experienced in intensive care, pulmonology, and emergency medicine and involved in the management of COVID19 patients (GC, YEC, and CP). To adjudicate each case, the expert panel was blinded to the results of FDG PET/CT but had full access to medical records, focusing on the kinetics of monitoring, treatment (notably oxygen delivery), and biology from 3 days before to 3 days after FDG PET/CT. Patients' short-term clinical outcome was classified as follows: worsening, stability, improvement. Further details are provided in the supplementary data online.

\section{Statistical analysis}

Categorical variables were collected as numbers $(n)$ and percentages (\%). Continuous variables were described as median and interquartile range (IQR). Correlation was computed using Spearman correlation analysis. Data were computed using Statistica for Windows (version 15.3). Wilcoxon signed-rank test were performed. A $p$ value $<0.05$ was considered significant.

\section{Results}

Fourteen patients were initially included in this study. One patient was secondarily excluded, as it retrospectively appeared that his FDG PET/CT has been performed out-of- time (day 22). The flowchart for patients' selection is presented in Fig. 1.

\section{Patients characteristics}

Clinical characteristics of patients on admission are presented in Table 1. Median age was 68 years (IQR 57-80), with a balanced proportion between men and women. Most patients had no underlying disorders, and the most common comorbidity was hypertension in six of thirteen patients (46\%), followed by prior cerebrovascular diseases in two of thirteen patients (15\%). Fever, irritating cough, and fatigue were the most common symptoms on admission, followed by dyspnea. Three of thirteen patients (23\%) had diarrhea, and rhinorrhea was the rarest symptom on admission. Laboratory findings on admission are summarized in Table 2. Eight of thirteen patients (61\%) showed lymphopenia (defined as lymphocyte count $<1.0 \times 10^{9} / \mathrm{L}$ ). All patients had a C-reactive protein level above the normal range (defined as $>5 \mathrm{mg} / \mathrm{L}$ ).

The median duration from the onset of symptoms to hospital admission was 5 days (IQR 4-7). The median duration from the onset of symptoms to FDG PET/CT was 10 days (IQR 9-12). During the time period including 3 days before and 3 days after the PET study, five patients (38\%) were classified as clinical worsening, one patient $(8 \%)$ as clinical stability, and seven patients (54\%) as clinical improvement; eleven patients $(85 \%)$ required oxygen therapy through nasal canula at low flow rates (1-9 L/min), none needed noninvasive mechanical ventilation, no patient experienced a secondary bacterial infection, none developed any acute cardiac or kidney injuries, none was coinfected with influenza A and B viruses, Mycoplasma pneumoniae, Streptococcus pneumoniae, and Legionella pneumophila, and none received corticosteroids. None of our patients had any clinical evidence for myocardial ischemia, neurological, or gastrointestinal involvement during this time period.

In the hospitalization phase up to the end of 3rd of May, none of our patients died, eleven patients $(85 \%)$ were discharged, five patients (38\%) required intensive care unit (ICU) care, and one patient ( $8 \%$ ) required invasive mechanical ventilation. Discharged patients had experienced a median of 8 days (IQR 7-13) of hospital stay.

\section{Chest CT findings (Table 3)}

Seven of thirteen patients (54\%) were classified as CT worsening, five of thirteen patients (38\%) as CT stability, and one of thirteen patients (8\%) as CT improvement. The median duration from the initial chest CT scan to the FDG PET/CT was 5 days (IQR 2-5). There was a tendency for the CT abnormalities extension to increase between the two chest CT scans (median 50\%; IQR 20-60 versus median 60\%; IQR $40-60, p=0.11$, Table E3 in the supplementary data 
Fig. 1 Study flow chart. Twentysix patients with laboratoryconfirmed COVID-19 were hospitalized at the Centre Hospitalier Princesse Grace of Monaco from March 27th to May 3rd, 2020. Twelve patients were excluded because they were admitted out of the selected time period from the onset of the symptoms or without chest CT ground-glass opacities or consolidation. One patient was secondary excluded because further elicitation retrospectively revealed the onset of symptoms occurred 22 days before FDG PET/ CT study

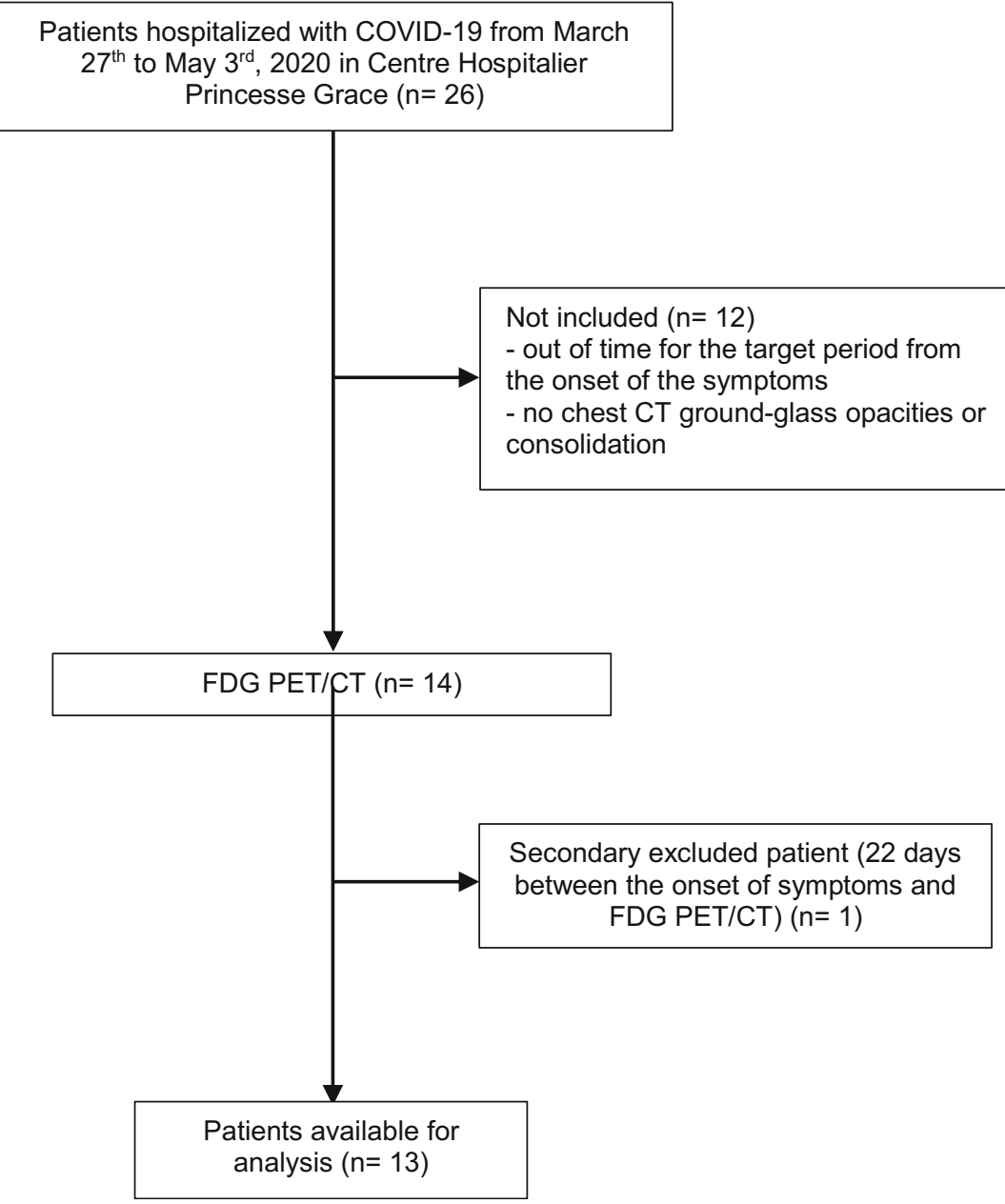

online). GGO appeared to be the first step, then focal consolidation occurred. Lower lobes were chronologically the first areas to exhibit lung consolidation. Only one patient had a unique lobe involvement (right lower lobe, Fig. E1 in the supplementary data online). No patient demonstrated any adenopathy on CT (defined as lymph node with short-axis dimension $\geq 10 \mathrm{~mm}$ ).

\section{FDG PET/CT findings (Table 4)}

FDG uptake for lungs GGO and consolidation areas was increased and heterogeneous, with $\mathrm{SUV}_{\max }$ ranging from 4.7 to 16.3 and hypermetabolic volumes ranging from 3.3 to $990 \mathrm{~mL}$. Three of thirteen patients (23\%) presented mild nasopharyngeal FDG uptake. Two of thirteen patients (15\%) had mild diffuse digestive FDG uptake and one of thirteen patients (8\%) had mild incidental diffuse FDG uptake in the gallbladder, without $\mathrm{CT}$ abnormalities. No patient had significant digestive focal or segmental FDG uptake abnormality. Two of thirteen patients (15\%) and five of thirteen patients (38\%) presented bone marrow or splenic FDG mild increased uptake respectively. All patients showed increased FDG uptake in mediastinal lymph nodes, and five of thirteen patients $(38 \%)$ showed increased FDG uptake in subclavicular lymph nodes.

We did not find any evidence for myocardial inflammation according to clinical symptoms, cardiac biomarkers, electrocardiography, or echocardiography. There was no significant physiological myocardial FDG uptake in any of the patients except one, despite the absence of a dedicated regimen designed for inducing myocardial extinction. Myocardial FDG uptake was sometimes below the circulating blood activity and appeared as a negative halo (see Fig. E2 in the supplementary data).

Relationship between FDG PET/CT inflammatory status, chest $\mathrm{CT}$ evolution, and short-term clinical outcome is summarized in Table 5. Overall, two of thirteen patients (15\%) were classified as having a low inflammatory profile and eleven of thirteen patients (85\%) as having an inflammatory profile. Figure 2 illustrates an inflammatory profile patient and Fig. 3 a low inflammatory profile patient. The two low inflammatory profile patients demonstrated clinical improvement. However, five of the eleven inflammatory profile patients (45\%) also clinically improved. All patients with 
Table 1 Patients' clinical characteristics on admission

\begin{tabular}{ll}
\hline & $n=13, n(\%)$ \\
\hline Age, groups & \\
$<65$ & $5(38)$ \\
$\geq 65$ & $8(61)$ \\
Gender & \\
Women & $6(46)$ \\
Men & $7(53)$ \\
Comorbidities & \\
Any & $7(53)$ \\
Hypertension & $6(46)$ \\
Prior cerebrovascular disease & $2(15)$ \\
Chronic kidney disease & $1(8)$ \\
Former or current smoking & $1(8)$ \\
Diabetes & $1(8)$ \\
Chronic obstructive pulmonary disease & 0 \\
Symptoms on admission & \\
Fatigue & $12(92)$ \\
Irritative cough & $12(92)$ \\
Fever & $12(92)$ \\
Dyspnea & $9(69)$ \\
Aguesia & $5(38)$ \\
Anosmia & $3(23)$ \\
Diarrhea & $3(23)$ \\
Rhinorrhea & $1(8)$ \\
\hline &
\end{tabular}

worsening CTs were inflammatory patients. No significant correlation was found between either overall extent of lung abnormalities on chest CT images or blood levels of Creactive protein on one hand, and lung $\mathrm{SUV}_{\max }$, lung hypermetabolic volumes, and mediastinal lymph nodes $\mathrm{SUV}_{\max }$ on the other hand.

Among the two patients with a mild, although abnormal, FDG uptake on bone marrow, one showed a short-term
Table 2 Selected biological results on admission

\begin{tabular}{ll}
\hline & $n=13$, median (IQR) \\
\hline C-reactive protein, mg/L & $69(35.8-105)$ \\
$\geq 5 \mathrm{mg} / \mathrm{L}, n(\%)$ & $13(100)$ \\
White blood cell count, $\times 10^{9} / \mathrm{L}$ & $5.83(4.56-7.73)$ \\
$>10 \times 109 / \mathrm{L}, n(\%)$ & $1(8)$ \\
$<4 \times 109 / \mathrm{L}, n(\%)$ & $2(15)$ \\
Neutrophil count, $\times 10^{9} / \mathrm{L}$ & $5.25(4.11-6.62)$ \\
Lymphocyte count, $\times 10^{9} / \mathrm{L}$ & $0.91(0.77-1.20)$ \\
$<1.0 \times 109 / \mathrm{L}, n(\%)$ & $8(61)$ \\
Eosinophil count, $\times 10^{9} / \mathrm{L}$ & $0(0-0.012)$ \\
Basophil count, $\times 10^{9} / \mathrm{L}$ & $0.012(0-0.016)$ \\
Hemoglobin, $\mathrm{g} / \mathrm{dL}$ & $13.6(12.6-15.5)$ \\
Platelet count, $\times 10^{9} / \mathrm{L}$ & $201(168-285)$ \\
AST, U/L & $36(30-46)$ \\
ALT, U/L & $23(18-40)$ \\
Urea, mmol/L & $6(5.2-8.3)$ \\
Creatinine, $\mu$ mol/L & $82(61-99)$ \\
PCT, ng/ml & $0.12(0.08-0.13)$ \\
NT-proBNP, pg/mL & $124(55-307)$ \\
Fibrinogen, g/L & $4.54(4.15-6.32)$ \\
$>4$ g/l, $n(\%)$ & $11(85)$ \\
D-dimer, $\mu \mathrm{g} / \mathrm{L}$ & $1.069(0.899-3.346)$ \\
High-sensitivity cardiac troponin $\mathrm{I}, \mathrm{ng} / \mathrm{mL}$ & $0.01(0.009-0.015)$ \\
\hline
\end{tabular}

$A S T$ aspartate aminotransferase, $A L T$ alanine transaminase, $P C T$ procalcitonin, NT-proBNP $\mathrm{N}$ terminal pro B type natriuretic peptide, $L D H$ lactate dehydrogenase

clinical improvement, while the other one showed a clinical worsening. Moreover, among the five patients with splenic FDG mild increased uptake, two were classified as having a short-term clinical improvement and three showed a shortterm clinical worsening. Patients with bone marrow or splenic FDG mild increased uptake did not have a higher body

Table 3 Chest CT findings

\begin{tabular}{lll}
\hline Number of patients (\%) & $\begin{array}{l}\text { Initial chest CT scan on } \\
\text { admission }\end{array}$ & $\begin{array}{l}\text { Second chest CT scan concomitant with } \\
\text { FDG PET/CT }\end{array}$ \\
\hline Ground-glass opacities & $13(100)$ & $13(100)$ \\
Consolidation & $7(54)$ & $13(100)$ \\
Subclavicular adenopathy* & 0 & 0 \\
Mediastinal adenopathy* & 0 & 0 \\
Pleural effusion & 0 & 0 \\
Linear opacities & $6(46)$ & $9(69)$ \\
Opacities with a cravy-paving pattern & $3(23)$ & $4(31)$ \\
Emphysema or fibrosis & 0 & 0 \\
Number of lobes affected, median (IQR) & $5(5-5)$ & $5(5-5)$ \\
Overall extent of lung involvement in & $50(20-60)$ & $60(40-60)$ \\
$\%$, median (IQR) & & \\
\hline
\end{tabular}

* Defined as lymph node with short-axis dimension $\geq 10 \mathrm{~mm}$ 
Table 4 FDG PET/CT findings

\begin{tabular}{ll}
\hline Increased FDG uptake, CT localization & Number of patients (\%) \\
\hline Subclavicular lymph node & $5(38)$ \\
Mediastinal lymph node, & $13(100)$ \\
Gallbladder & $1(8)$ \\
Nasopharynx & $3(23)$ \\
Bone marrow & $2(15)$ \\
Spleen & $5(38)$ \\
Brain focal abnormalities & 0 \\
Digestive tube, focal or segmental & 0 \\
Myocardium, pathological & 0 \\
Myocardium, physiological & $1(8)$ \\
\hline
\end{tabular}

temperature than the other patients (bone marrow FDG uptake group median $38^{\circ} \mathrm{C}$; IQR $37-39$ versus median $37.3^{\circ} \mathrm{C}$; IQR $37-38, p=0.66$; splenic FDG uptake group median $37.3^{\circ} \mathrm{C}$; IQR 37-38.5 versus median 37; IQR 37-37.75, $p=0.76$ ).

\section{Discussion}

Imaging of inflammatory process in patients with COVID-19 at the presumed peak of the disease using FDG PET/CT has not been previously performed. Our main result is that COVID-19 patients presenting GGO or consolidation on initial chest CT demonstrate a lung inflammation at the presumed peak of the inflammatory phase that varies widely from minor to extensive involvement. Our results suggest that tropism of this inflammatory process is mostly focused on the lower respiratory tract. However, we were unable to correlate this inflammation to chest CT evolution or shortterm clinical outcome.
The increased and heterogeneous FDG uptake we observed in lungs probably results from an intense activation of inflammatory cells. Glycolytic activity in human lymphocytes has been known for decades and FDG PET/CT has a high sensitivity to evidence lymphocyte activation [10, 16, 17]. The exact contribution of specific immune cells activated in lung parenchyma during COVID-19 cannot be deducted from FDG PET/CT data alone as increasing anaerobic glycolysis is also described in activate inflammatory cells such as monocytes and granulocytes [10]. However, intra-alveolar neutrophilic infiltration found in medical autopsies is rather reported during secondary bacterial infection [18]. Intra-alveolar infiltration by mononuclear inflammatory cells is therefore the most likely phenomenon explaining FDG pathological uptake. The good clinical tolerance to this infiltration, at least at the beginning of the inflammatory phase, has been observed in other disorders such as lymphoma or sarcoidosis [19]. In COVID 19, Gattinoni et al. [20] reported two groups of patients at the critical phase: those with a preserved pulmonary compliance (type L) who do not behave as classical ARDS, and those with an impaired pulmonary compliance (type $\mathrm{H}$ ) with typical ARDS behavior and prognosis. It is likely that our patients belonged to the first group, with a better prognosis.

Lung changes on chest $\mathrm{CT}$ scan were consistent with other studies [8, 9, 15]. As previously reported, consolidation occurred after GGO and lung abnormality involvement reached a maximum on the second chest CT scan for follow-up concomitant with the FDG PET/CT scan. We found neither pleural effusion nor thoracic lymphadenopathy, which are known as uncommon findings in COVID-19 [8]. Despite the absence of abnormal CT criteria for thoracic lymphadenopathy, FDG PET/CT demonstrated mediastinal and subclavicular lymph node activation which was consistent with the few other reported cases [21-24]. Angiotensin-converting enzyme 2 is a functional receptor for SARS-Cov [25]. Lymph nodes FDG uptake is probably linked to cytokine-mediated inflammation
Table 5 Relationship between FDG PET/CT inflammatory status, chest $\mathrm{CT}$ evolution and shortterm clinical outcome

\begin{tabular}{|c|c|c|c|c|}
\hline & & \multicolumn{3}{|c|}{ Short-term clinical outcome } \\
\hline & & Worsening & Stability & Improvement \\
\hline \multirow[t]{3}{*}{ Chest CT evolution } & Worsening $(n=7)$ & 4 & 0 & 3 \\
\hline & Stability $(n=5)$ & 1 & 1 & 3 \\
\hline & Improvement $(n=1)$ & 0 & 0 & 1 \\
\hline \multirow{2}{*}{$\begin{array}{l}\text { Inflammatory status FDG } \\
\text { PET/CT }\end{array}$} & Inflammatory profile $(n=11)$ & 5 & 1 & 5 \\
\hline & $\begin{array}{l}\text { Low inflammatory profile } \\
\qquad(n=2)\end{array}$ & 0 & 0 & 2 \\
\hline \multicolumn{2}{|c|}{ Inflammatory profile FDG PET/CT and CT worsening $(n=7)$} & 4 & 0 & 3 \\
\hline \multicolumn{2}{|c|}{$\begin{array}{l}\text { Low inflammatory profile FDG PET/CT and CT } \\
\text { improvement/stability }(n=2)\end{array}$} & 0 & 0 & 2 \\
\hline \multicolumn{2}{|c|}{$\begin{array}{l}\text { Inflammatory profile FDG PET/CT and CT improvement/stability } \\
(n=4)\end{array}$} & 1 & 0 & 3 \\
\hline
\end{tabular}


Fig. 2 A 53-year-old man patient with moderate respiratory symptoms referred for FDG PET/CT at day 14 after the onset of symptoms. Images showed multiple peripheral and bilateral groundglass opacities with intense increased FDG uptake $\left(\mathrm{SUV}_{\max }\right.$ 16.3). The following day, respiratory status dramatically improved without need of supplemental oxygen, and patient was discharged from hospital 3 days later. Left: A CT transverse slice, B FDG PET slice, and C FDG PET and CT-fused images. Right: whole-body maximal intensity projection (MIP) image, displaying also mediastinal lymph nodes FDG uptake
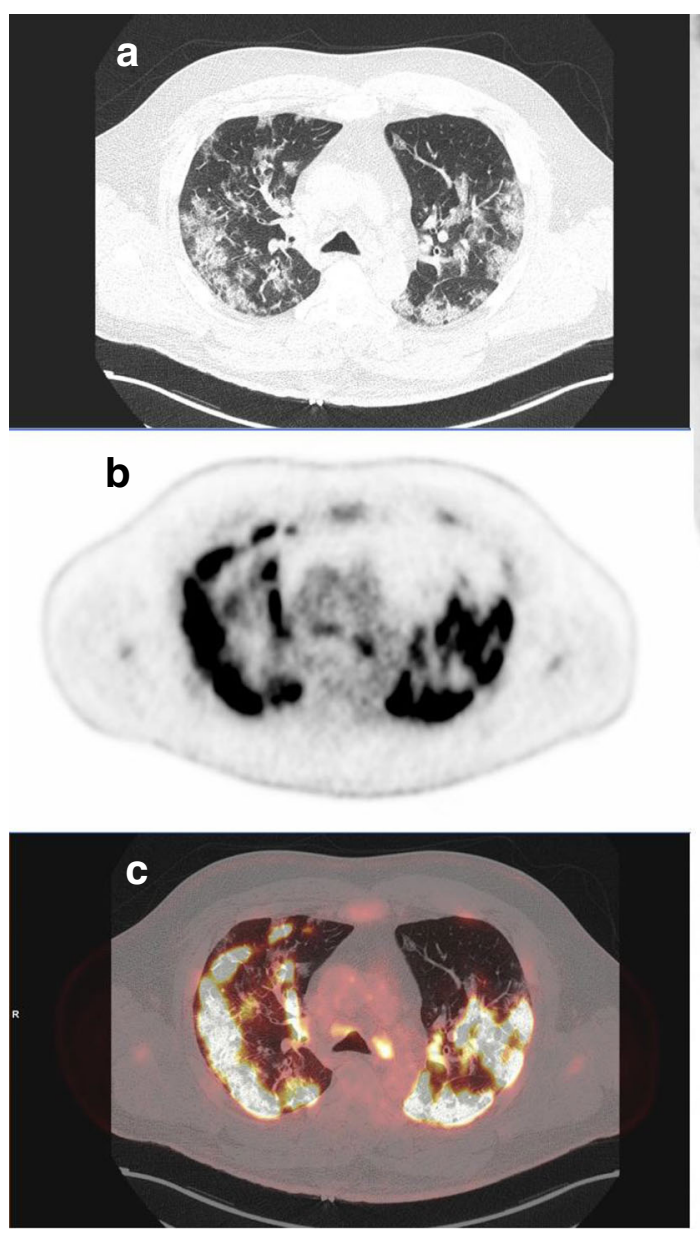

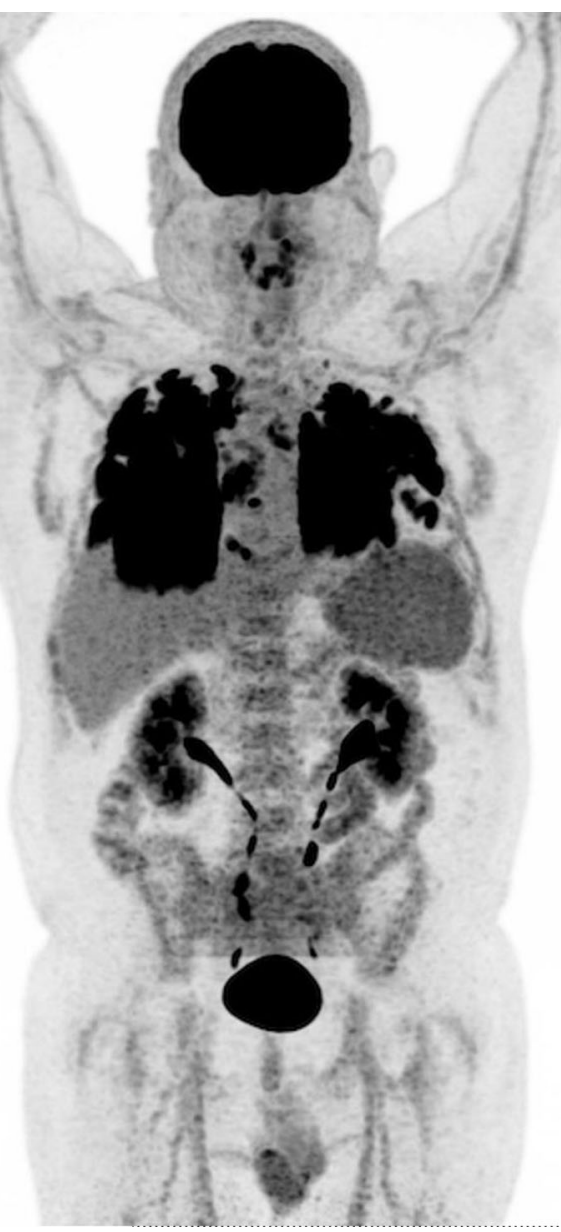

rather than to direct viral infection, considering the absence of angiotensin-converting enzyme 2 receptors (ACE2) on hemato-lymphoid cells [26].

The mildly increased FDG uptake observed in the spleen and bone marrow in some patients might be in response to an inflammatory condition [27]. Clinical short-term outcome of these patients was similar to that of other patients. However, larger series will be required to elucidate if an increased spleen or bone marrow FDG uptake represents an early sign of severe systemic inflammatory response and could be associated with clinical outcome in COVID-19.

Surprisingly, in all patients except one, no significant physiological myocardial FDG uptake was observed, despite the lack of any specific preparation aiming to suppress physiologic myocardial glucose metabolism. We did not find any explanation for this, suggesting that during COVID-19 infection, the myocardial metabolic pathway toward glycolysis is not (or weakly) activated. It also suggests that catecholaminergic stimulation increasing myocardial glucose uptake [28] was not present in almost all our patients.

Only few extra-pulmonary hotspots were found among our population although FDG PET/CT should be sensitive enough for detecting extra-pulmonary lesions in COVID19. This is consistent with the fact that none of our patients had clinical or biological manifestations of extrapulmonary disease at the time of the PET study. This may be explained by our eligibility criteria, especially $1 /$ excluding patients under mechanical ventilation, therefore discarding the most severe patients and 2/ with a time period from day 6 to day 14 of the onset of symptoms, when some minor non-pulmonary manifestations could have already disappeared.

Despite close geographical vicinity to Northern Italy, which was the second most affected country in the world at the beginning of the study in March 27th, 2020, Monaco has been poorly affected by COVID-19 during the study period. For evident safety reasons, we did not include patients with mechanical-assisted ventilation. Therefore, our population is a subset of non-critically ill patients. All patients were nevertheless consecutively enrolled, and their images are probably representative of the rate and the extent of lung inflammation. The limited statistical power may explain some of our results, especially the absence of significant relationship between biological markers of inflammation and lung FDG uptake. 
Fig. 3 A 55-year-old man patient who presented severe respiratory symptoms in the days before FDG $\mathrm{PET} / \mathrm{CT}$. Images showed bilateral consolidation with mild increased FDG uptake $\left(\mathrm{SUV}_{\max } 5\right)$. Despite this mild inflammation, the patient remained symptomatic for days requiring oxygen therapy through nasal canula at low flow rates and was discharged from hospital only 13 days later. Left: A CT transverse slice, B FDG PET slice, and $\mathbf{C}$ FDG PET and CT-fused images. Right: wholebody MIP image demonstrating that FDG uptake in mediastinal lymph nodes is above lung FDG uptake (respectively, $\mathrm{SUV}_{\max } 6.9$ versus 5)

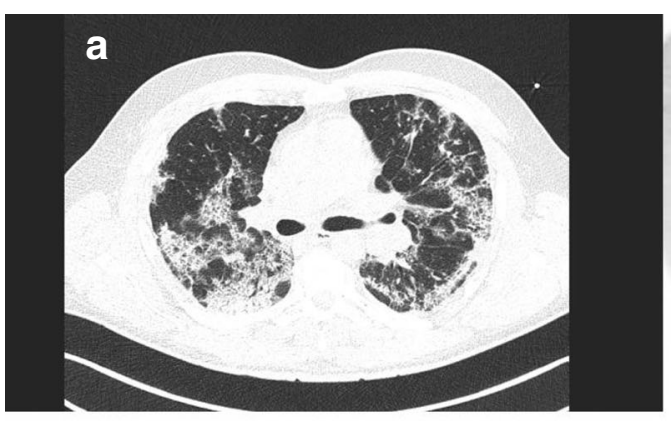

b
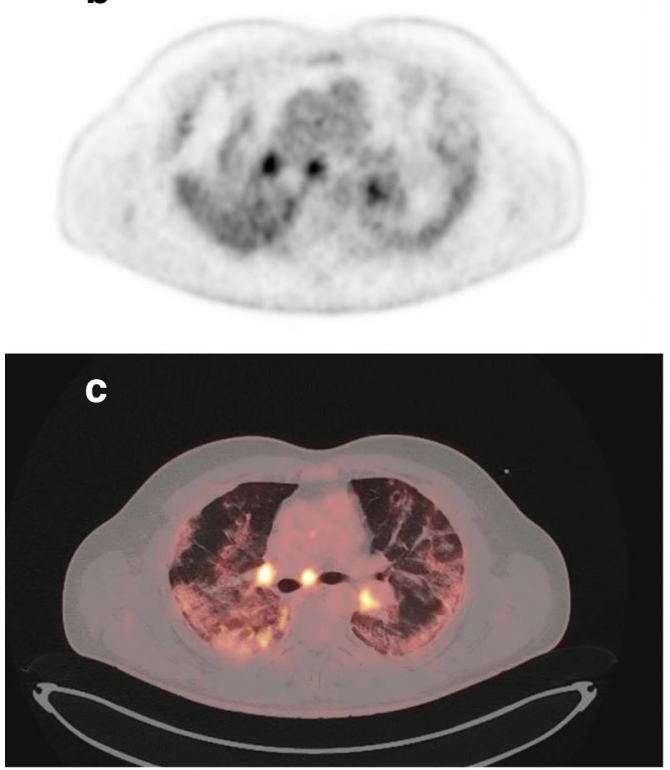

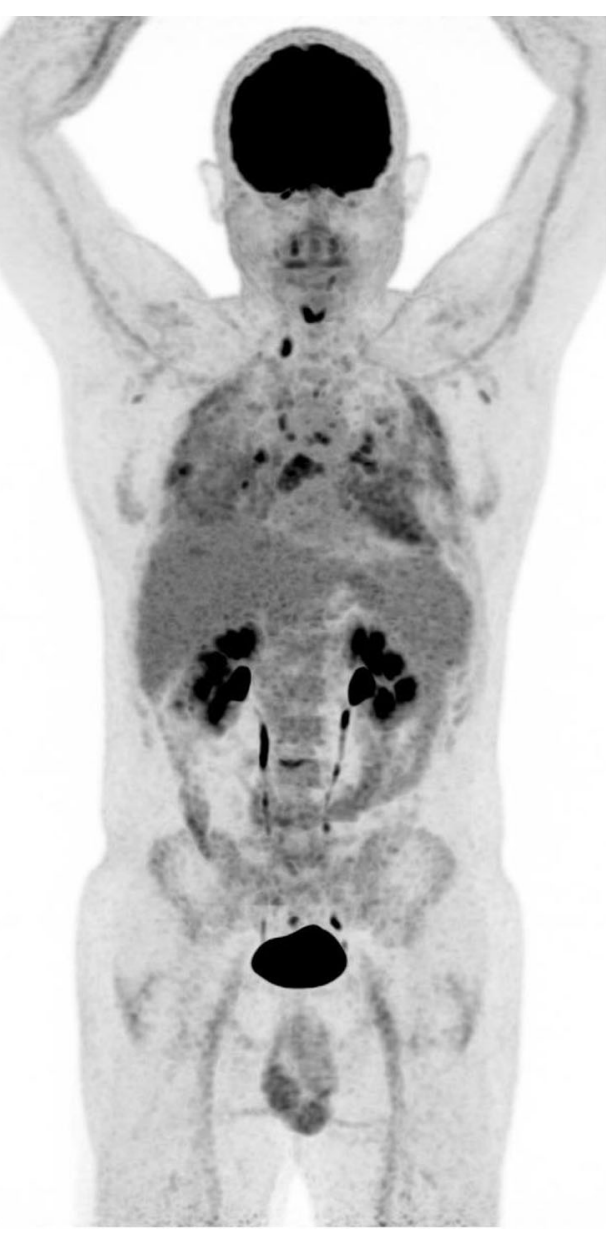

A prolonged follow-up is required to determine if a relationship exists between initial lung inflammation and longterm sequelae such as residual lung fibrosis and respiratory failure. Therefore, further studies comprising late CT images and functional respiratory examination will be required. However, thanks to a high sensitivity, FDG PET/CT may be a useful imaging method for a comprehensive understanding of the pathogenesis underlying COVID-19 inflammatory process.

Acknowledgments We would like to thank all staff at the dedicated COVID unit and the Nuclear Medicine Department staff of Centre Hospitalier Princesse Grace for their efforts to make this study possible.

We acknowledge all healthcare staff who are on the frontline of caring for patients.

We thank Dr. Jérémie Tordo for his contributions to the revision.

Availability of data and material The datasets generated during and/or analyzed during the current study are available from the corresponding author on reasonable request.

Authors' contributions MF, GC, YEC, and CP designed the study and enrolled the patients. MF supervised the study. MD, GC, YEC, RF, IR, $\mathrm{BS}, \mathrm{VN}, \mathrm{FH}, \mathrm{BP}, \mathrm{FB}, \mathrm{OKP}, \mathrm{FG}, \mathrm{CP}$, and MF contributed to acquisition of clinical, biological, and imaging data. $\mathrm{MF}, \mathrm{MD}$, and $\mathrm{FB}$ performed statistical analysis. MD and MF wrote the manuscript. GC, YEC, RF, OKP,
FG, and CP contributed to critical revision of the report. All authors approved the final draft of the manuscript.

\section{Compliance with ethical standards}

Conflict of interest The authors declare that they have no conflict of interest.

Ethics approval All procedures performed in studies involving human participants were in accordance with the ethical standards of the institutional and/or national research committee and with the 1964 Helsinki declaration and its later amendments or comparable ethical standards. "Comité de Pilotage de la recherche du Centre Hospitalier Princesse Grace" (Pilot committee for research of Centre Hospitalier Princesse Grace) was the institutional review board that approved the study.

Informed consent Informed consent was obtained from all individual participants included in the study.

\section{References}

1. Huang C, Wang Y, Li X, Ren L, Zhao J, Hu Y, et al. Clinical features of patients infected with 2019 novel coronavirus in Wuhan, China. Lancet (London, England). 2020;395(10223): 497-506. 
2. Wang D, Hu B, Hu C, Zhu F, Liu X, Zhang J, et al. Clinical characteristics of 138 hospitalized patients with 2019 novel coronavirus-infected pneumonia in Wuhan, China. JAMA. 2020;323(11):1061-9.

3. Du Y, Tu L, Zhu P, Mu M, Wang R, Yang P, et al. Clinical features of 85 fatal cases of COVID-19 from Wuhan: a retrospective observational study. Am J Respir Crit Care Med. 2020. https://doi.org/ 10.1164/rccm.202003-0543OC.

4. Zheng S, Fan J, Yu F, Feng B, Lou B, Zou Q, et al. Viral load dynamics and disease severity in patients infected with SARSCoV-2 in Zhejiang province, China, January-March 2020. BMJ. 2020;369:m1443.

5. Lee E, Ng MY, Khong PL. COVID-19 pneumonia: what has CT taught us? Lancet Infect Dis. 2020;20(4):384-5.

6. Li Y, Yao L, Li J, Chen L, Song Y, Cai Z, et al. Stability issues of RT-PCR testing of SARS-CoV-2 for hospitalized patients clinically diagnosed with COVID-19. J Med Virol. 2020. https://doi.org/10. 1002/jmv. 25786.

7. Leonard-Lorant I, Delabranche X, Severac F, Helms J, Pauzet C, Collange O, et al. Acute pulmonary embolism in COVID-19 patients on CT angiography and relationship to D-dimer levels. Radiology. 2020:201561. https://doi.org/10.1148/radiol. 2020201561.

8. Xu X, Yu C, Qu J, Zhang L, Jiang S, Huang D, et al. Imaging and clinical features of patients with 2019 novel coronavirus SARSCoV-2. Eur J Nucl Med Mol Imaging. 2020;47(5):1275-80.

9. Ding X, Xu J, Zhou J, Long Q. Chest CT findings of COVID-19 pneumonia by duration of symptoms. Eur J Radiol. 2020;127: 109009. https://doi.org/10.1016/j.ejrad.2020.109009.

10. de Prost N, Tucci MR, Melo MF. Assessment of lung inflammation with 18F-FDG PET during acute lung injury. AJR Am J Roentgenol. 2010;195(2):292-300.

11. Chefer S, Thomasson D, Seidel J, Reba RC, Bohannon JK, Lackemeyer MG, et al. Modeling [(18)F]-FDG lymphoid tissue kinetics to characterize nonhuman primate immune response to Middle East respiratory syndrome-coronavirus aerosol challenge. EJNMMI Res. 2015;5(1):65.

12. Chen DL, Bedient TJ, Kozlowski J, Rosenbluth DB, Isakow W, Ferkol TW, et al. [18F] fluorodeoxyglucose positron emission tomography for lung antiinflammatory response evaluation. Am J Respir Crit Care Med. 2009;180(6):533-9.

13. WHO. Clinical management of severe acute respiratory infection when novel coronavirus (nCoV) infection is suspected: interim guidance, 2020. Published January 25, 2020. Accessed Mar 20, 2020. https://apps.who.int/iris/handle/10665/330854

14. Paez D, Gnanasegaran G, Fanti S, Bomanji J, Hacker M, Sathkge $\mathrm{M}$, et al. COVID-19 pandemic: guidance for nuclear medicine departments. Eur J Nucl Med Mol Imaging. 2020. https://doi.org/10. 1007/s00259-020-04825-8.

15. Bernheim A, Mei X, Huang M, Yang Y, Fayad ZA, Zhang N, et al. Chest CT findings in coronavirus disease-19 (COVID-19): relationship to duration of infection. Radiology. 2020:200463. https://doi.org/10.1148/radiol.2020200463.

16. Marjanovic S, Skog S, Heiden T, Tribukait B, Nelson BD. Expression of glycolytic isoenzymes in activated human peripheral lymphocytes: cell cycle analysis using flow cytometry. Exp Cell Res. 1991;193:425-31.

17. Ishimori $T$, Saga $T$, Mamede M, Kobayashi H, Higashi $T$, Nakamoto Y, et al. Increased $18 \mathrm{~F}-\mathrm{FDG}$ uptake in a model of inflammation: concanavalin A-mediated lymphocyte activation. J Nucl Med. 2002;43:658-63.

18. Tian S, Xiong Y, Liu H, Niu L, Guo J, Liao M, et al. Pathological study of the 2019 novel coronavirus disease (COVID-19) through postmortem core biopsies. Mod Pathol. 2020:1-8.

19. Spagnolo P, Rossi G, Trisolini R, Sverzellati N, Baughman RP, Wells AU. Pulmonary sarcoidosis. Lancet Respir Med. 2018;6(5): 389-402.

20. Gattinoni L, Chiumello D, Caironi P, Busana M, Romitti F, Brazzi L, et al. COVID-19 pneumonia: different respiratory treatments for different phenotypes? Intensive Care Med. 2020:1-4.

21. Setti L, Kirienko M, Dalto SC, Bonacina M, Bombardieri E. FDGPET/CT findings highly suspicious for COVID-19 in an Italian case series of asymptomatic patients. Eur J Nucl Med Mol Imaging. 2020. https://doi.org/10.1007/s00259-020-04819-6.

22. Zou S, Zhu X. FDG PET/CT of COVID-19. Radiology. 2020: 200770. https://doi.org/10.1148/radiol.2020200770.

23. Qin C, Liu F, Yen TC, Lan X. 18F-FDG PET/CT findings of COVID-19: a series of four highly suspected cases. Eur J Nucl Med Mol Imaging. 2020;47:1281-6.

24. Polverari G, Arena V, Ceci F, Pelosi E, Ianniello A, Poli E, et al. $18 \mathrm{~F}$-fluorodeoxyglucose uptake in patient with asymptomatic severe acute respiratory syndrome coronavirus 2 (coronavirus disease 2019) Referred to positron emission tomography/computed tomography for NSCLC restaging. J Thorac Oncol. 2020;0. https://doi. org/10.1016/j.jtho.2020.03.022.

25. Li W, Moore MJ, Vasilieva N, Sui J, Wong SK, Berne MA, et al. Angiotensin-converting enzyme 2 is a functional receptor for the SARS coronavirus. Nature. 2003;426(6965):450-4.

26. Hamming I, Timens W, Bulthuis ML, Lely AT, Navis G, van Goor $\mathrm{H}$. Tissue distribution of ACE2 protein, the functional receptor for SARS coronavirus. A first step in understanding SARS pathogenesis. J Pathol. 2004;203(2):631-7.

27. Ahn SS, Hwang SH, Jung SM, Lee SW, Park YB, Yun M, et al. Evaluation of spleen glucose metabolism using ${ }^{18}$ F-FDG PET/CT in patients with febrile autoimmune disease. J Nucl Med. 2017;58(3):507-13.

28. Depre C, Vanoverschelde JL, Taegtmeyer H. Glucose for the heart. Circulation. 1999;99:578-88.

Publisher's note Springer Nature remains neutral with regard to jurisdictional claims in published maps and institutional affiliations. 